\title{
Distributed Power Allocation Game for Uplink OFDM Systems
}

\author{
Gaoning He, Sophie Gault \\ Motorola Labs \\ 91193 Gif-sur-Yvette - FRANCE \\ Email: gaoninghe@motorola.com \\ sophie.gault@motorola.com
}

\author{
Merouane Debbah \\ Supélec \\ 91192 Gif-sur-Yvette - FRANCE \\ Email: merouane.debbah@supelec.fr
}

\author{
Eitan Altman \\ INRIA \\ 06902 Sophia Antipolis - FRANCE \\ Email: eitan.altman@sophia.inria.fr
}

\begin{abstract}
In this paper, we consider the uplink of a single cell network with $K$ users simultaneously communicating with a base station using OFDM modulation over $N$ carriers. In such a scenario, users can decide their power allocation based on three possible Channel State Information (CSI) levels, which are called complete, partial and statistical. The optimal solutions for maximizing the average capacity with complete and statistical knowledge are known to be the water-filling game and the uniform power allocation respectively. We study the problem in the partial knowledge case. We formulate it as a strategy game, where each player (user) selfishly maximizes his own average capacity. The information structure that we consider is such that each player, at each time instant, knows his own channel state, but does not know the states of other players. We study the existence and uniqueness of Nash equilibrium. We find the optimal solution for the symmetric game considering two positive channel states, and we show the optimization problem for any $L$ states.
\end{abstract}

\section{INTRODUCTION}

The extension of OFDM [1] to allow simultaneous communication with multiple users is called OFDMA (Orthogonal Frequency Division Multiple Access) [2]. For this setting, efficient scheduling algorithms to optimize the users' rate exist and are based on multi-user diversity schemes [3] (only users with the best carrier to noise ratio (CNR) conditions access the network). This procedure is centralized: the scheduler (generally the base station) assigns to each mobile the carriers it is allowed to use. It requires an estimation by the scheduler of the $N$ carriers of the $K$ users, and hence an important feedback load. However, the uplink and downlink signalling used in the estimation do not only consume system resource but also increase the complexity. Moreover, for high mobility, the channel conditions vary and the algorithm becomes inaccurate. To reduce the feedback load, selective multiuser diversity algorithms have been introduced: only the users that have a CNR above a threshold send feedback to the scheduler [4] . Multiple feedback thresholds can be used [5] and are generally found numerically.

A way to avoid the constraints of a centralized procedure is to implement a decentralized one. Unlike centralized procedure, few signalling consumption is the main advantage of a decentralized scheme. Indeed, centralized schemes generally require complete information (all channels of all users) whereas in a decentralized one, each user is able to make decision based on partial information (e.g. only his own channel information), and this knowledge can be easily obtained by using few downlink signalling (e.g. a downlink training sequence). The potential gain of a decentralized procedure therefore lies in the fact that we can get rid of the signalling needed for a user to obtain other users' channel information, while a great amount of signalling is needed in a centralized system to get all users' information. Especially when the number of users increases or when the channel rapidly changes, this signalling consumption becomes non-negligible or even unacceptable. A natural framework to model decentralized schemes where users interact is game theory, which studies competition (as well as cooperation) between independent actors. Tools of game theory have already been frequently used as a central framework for modeling competition and cooperation in networking, see for example [6] and references therein.

In a fading multiple-access channel context, a game theoretic framework has been used in [7]. Users compete with rates as utility and powers as moves in the game, in what the authors call the water-filling game. They show that the unique Nash equilibrium in this game corresponds to the maximum Sum-Rate point of the capacity region. [8]. However, their results rely on the fact that each user has a nearly complete knowledge of the system (at least the knowledge of global interference and the condition to apply water-filling), and in particular, perfect channel state information (CSI) of all users in the cell. This is a necessary requirement in order to use the theory of games with complete information, and an usual assumption in many papers in the field, as the authors point out. Nevertheless, it is rarely possible in practice and one can usually only satisfy at best the requirements knowing only its own channel.

Power allocation problem has to be reconsidered with the assumption that one user only knows its own channel. In this paper, we show that power allocation problem can be modelled as a strategic non-cooperative game where each user only has partial knowledge and finds power levels maximizing his own utility. We study the existence of Nash equilibria in such context. We analyze the feasibility and practicability of applying decentralized schemes. Our main objective is to design a decentralized power allocation scheme modelled as 


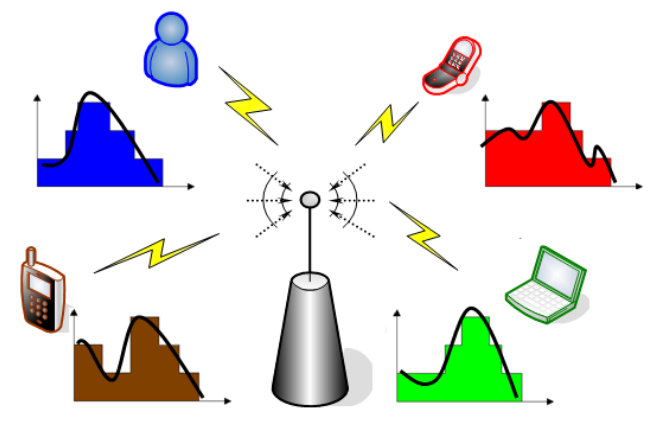

Fig. 1. Illustration of the uplink an OFDM system

a strategic game under some reasonable assumptions.

The paper is organized in the following form: the system model is introduced in section II. In section III, we present the problem and its solution when complete and statistic information is considered at the transmitter. In section IV, we provide a strategy game model to solve the problem with partial information. Finally, numerical results are provided in section $\mathrm{V}$ followed by conclusions in section VI.

\section{SYSTEM MODEL}

We consider a flat-fading multiple access channel (MAC) in a single cell network, where $K$ users are simultaneously communicating with a base station using OFDM modulation over $N$ carriers. At each time instant, each carrier $i$ of each user $k$ is characterized by a fading coefficient $h_{k}^{(i)}, k=$ $1, \ldots, K, i=1, \ldots, N$. It is distributed according to some (known) distribution function. We will denote by $\mathbf{H}$ a random variable having this distribution.

In the context, users have to decide how to allocate their power across time and subcarriers, based on some knowledge and information they may have, which may be complete, partial or only statistical CSI. More precisely, complete CSI means that, at each time instant, one user knows his fading as well as others' fadings; partial CSI means that one user knows only his fading and in the third case, each user only has a statistical knowledge of all channels. In this paper, we focus on the case of partial CSI, and we always assume that users have at least statistical knowledge of the fading and know the power budgets of the other users, which is expressed as

$$
\sum_{i=1}^{N} \mathbb{E}_{\mathbf{H}}\left[p_{k}^{(i)}\right] \leq \bar{P}_{k}
$$

On each carrier $i$, user $k$ sends the information $x_{k}^{(i)}=$ $\sqrt{p_{k}^{(i)}} s_{k}^{(i)}$, where $s_{k}^{(i)}$ is the transmitted data such as $\mathbb{E}\left[\left|s_{k}^{(i)}\right|^{2}\right]=1$. Note that a set $\mathbb{M}_{i} \subseteq\{1, \ldots, K\}$ of users can select the same frequency carrier $i$, which introduces interference. As a consequence, the received signal on carrier $i$ at the base station is given by:

$$
y^{(i)}=\sum_{k \in \mathbb{M}_{i}} h_{k}^{(i)} x_{k}^{(i)}+n^{(i)}
$$

where $n^{(i)}$ is a zero mean Gaussian noise with variance $\sigma^{2}$.

At the base station, the SINR of user $k$ on carrier $i$ is therefore:

$$
\operatorname{SINR}_{k}^{(i)}=\frac{p_{k}^{(i)} h_{k}^{(i)}}{\sigma^{2}+\sum_{\substack{j=1 \\ j \neq k}}^{K} p_{j}^{(i)} h_{j}^{(i)}}
$$

The corresponding ergodic capacity of user $k$ is given by:

$$
C_{k}=\mathbb{E}_{\mathbf{H}}\left[\sum_{i=1}^{N} \log _{2}\left(1+\frac{p_{k}^{(i)} h_{k}^{(i)}}{\sigma^{2}+\sum_{\substack{j=1 \\ j \neq k}}^{K} p_{j}^{(i)} h_{j}^{(i)}}\right)\right] .
$$

In a game theoretic context, the purpose of each user is to maximize selfishly its capacity.

\section{BACKGROUND}

First, we present the existing solutions correspond to the two scenarios: complete and statistical knowledge at the transmitter that we mentioned above, and are also inherently associated to particular implementation. We show that the complete knowledge scheme requires a centralized (or semidistributed) implementation whereas the other two (partial and statistical) can be implemented in a fully decentralized way. For simplicity of presentation, we consider a single cell network with two users simultaneously communicating with a base station in a single carrier setting. Each user $k=1,2$ chooses a strategy $p_{k} \in \mathbb{R}_{+}$.

\section{A. Complete knowledge at Tx}

With complete CSI, the case of a single carrier is studied in the first part of [7]. Let us denote by $p_{1}\left(h_{1}, h_{2}\right)$ and $p_{2}\left(h_{1}, h_{2}\right)$ the strategies of the two users. For a fixed strategy $p_{2}\left(h_{1}, h_{2}\right)$, finding the optimal strategy $p_{1}\left(h_{1}, h_{2}\right)$ for user 1 requires solving the following optimization problem

$$
\begin{array}{ll}
\max C_{1}=\max _{p_{1}\left(h_{1}, h_{2}\right)} & \mathbb{E}_{h_{1}, h_{2}}\left[\log _{2}\left(1+\frac{p_{1}\left(h_{1}, h_{2}\right) h_{1}}{\sigma^{2}+p_{2}\left(h_{1}, h_{2}\right) h_{2}}\right)\right] \\
\text { s.t. } & \mathbb{E}_{h_{1}, h_{2}}\left[p_{1}\left(h_{1}, h_{2}\right)\right] \leq \bar{P}_{1} \\
& p_{1}\left(h_{1}, h_{2}\right) \geq 0
\end{array}
$$

and similarly for the second user. The solution of the optimization problem is the waterfilling power allocation [9]

$$
p_{1}\left(h_{1}, h_{2}\right)=\left(\lambda_{1}-\frac{\sigma^{2}+p_{2}\left(h_{1}, h_{2}\right) h_{2}}{h_{1}}\right)^{+}
$$

where $(x)^{+}=\max \{x, 0\}$ and $\lambda_{1}$ is chosen in order to satisfy the constraint

$$
\mathbb{E}_{h_{1}, h_{2}}\left(\lambda_{1}-\frac{\sigma^{2}+p_{2}\left(h_{1}, h_{2}\right) h_{2}}{h_{1}}\right)^{+}=\bar{P}_{1} .
$$

Note that the solution (6) depends on the power allocation of the second user, which the first user does not know, and reciprocally for the second user. However, given the game model, each user is able to adjust his strategy adaptively to its guess of the strategy of the other user. In [7], the authors show that this process converges to a unique equilibrium and that it is time-sharing (i.e., for a given realization of the fading, the 
optimal $p_{1}\left(h_{1}, h_{2}\right)$ and $p_{2}\left(h_{1}, h_{2}\right)$ can not be simultaneously strictly positive). It corresponds to the maximum Sum-Rate point of the capacity region:

$$
\left\{\begin{array}{l}
p_{1}\left(h_{1}, h_{2}\right)=\left(\lambda_{1}-\frac{\sigma^{2}}{h_{1}}\right)^{+}, \text {when } h_{1} \geq \frac{\lambda_{2}}{\lambda_{1}} h_{2} \\
p_{2}\left(h_{1}, h_{2}\right)=\left(\lambda_{2}-\frac{\sigma^{2}}{h_{2}}\right)^{+}, \text {when } h_{2} \geq \frac{\lambda_{1}}{\lambda_{2}} h_{1} .
\end{array}\right.
$$

with $p_{1}\left(h_{1}, h_{2}\right)=0$ and $p_{1}\left(h_{1}, h_{2}\right)=0$ in other cases. The waterfilling levels are obtained by solving the system of equations

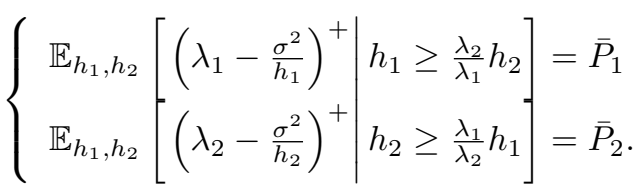

Thus, an interesting conclusion is that the result of the selfish behavior of the users will result in the joint optimization of the global capacity of the channel.

However, in (8), there exists two conditions to indicate users whether they should transmit power or not. For instance, the water-filling condition for user 1 is: $h_{1} \geq \frac{\lambda_{2}}{\lambda_{1}} h_{2}$, where $\lambda_{1}$ and $\lambda_{2}$ are both known to user 1 (one can find in (9) that $\lambda_{1}$ and $\lambda_{2}$ only depend on the distribution of $h_{1}$ and $h_{2}$ ), but $h_{2}$ (the channel realization of user 2) may not be known by user 1 . In practice, for such a scenario with multiple users (e.g. uplink of multi-user OFDM), a certain amount of UL/DL signallings are needed to be exchanged between the base station and users so that each user can obtain the information for this condition. However, when the number of users is large, the corresponding amount of signalling will dramatically reduce the global throughput and become unacceptable for a real system. It is for this reason that we are motivated to investigate a novel power allocation scheme such that each user is able to decide and allocate his power without knowing the channel states of others, therefore, the UL/DL signalling mentioned above are not required any more.

\section{B. Statistical knowledge at $T x$}

When users have only statistical knowledge of their channel, as well as of the channels of other users, the strategies of the two users $p_{1}$ and $p_{2}$ can not depend on the realization of the fading and are necessarily fixed. For a fixed strategy $p_{2}$, finding the optimal strategy $p_{1}$ for user 1 requires solving the following optimization problem

$$
\begin{aligned}
\max C_{1}=\max _{p_{1}} & \mathbb{E}_{h_{1}, h_{2}}\left[\log _{2}\left(1+\frac{p_{1} h_{1}}{\sigma^{2}+p_{2} h_{2}}\right)\right] \\
\text { s.t. } & p_{1} \leq \bar{P}_{1} \\
& p_{1} \geq 0
\end{aligned}
$$

and similarly for the second user. Since the function to maximize is an increasing function of $p_{1}$, the solution of the optimization problem (10) is obviously:

$$
p_{1}=\bar{P}_{1}
$$

and similarly for the second user $p_{2}=\bar{P}_{2}$. Since the users do not have any information about their channel, the best they can do is always transmit at the maximal constant power they can afford. This results in (time domain) uniform power allocation.

\section{Distributed Power Allocation Game}

Here, we consider a distributed power allocation scheme with partial knowledge at the transmitter. To be precise, at each time instant, each user knows the realization of its own channel and the statistical knowledge of other user's channel. In this case, $p_{1}$ can only depend on $h_{1}$ and $p_{2}$ on $h_{2}$. Let us denote by $p_{1}\left(h_{1}\right)$ and $p_{2}\left(h_{2}\right)$ the strategies of the two users. For a fixed strategy $p_{2}\left(h_{2}\right)$, finding the optimal strategy $p_{1}\left(h_{1}\right)$ for user 1 requires to solve the following optimization problem

$$
\begin{aligned}
& \max C_{1}=\max _{p_{1}\left(h_{1}\right)} \mathbb{E}_{h_{1}, h_{2}}\left[\log _{2}\left(1+\frac{p_{1}\left(h_{1}\right) h_{1}}{\sigma^{2}+p_{2}\left(h_{2}\right) h_{2}}\right)\right] \\
& \text { s.t. } \quad \mathbb{E}_{h_{1}}\left[p_{1}\left(h_{1}\right)\right] \leq \bar{P}_{1} \\
& p_{1}\left(h_{1}\right) \geq 0
\end{aligned}
$$

and similarly for the second user. Note that the term $p_{2}\left(h_{2}\right)$ is unknown to the first user and that its strategy can not depend on the particular realization of $h_{2}$. User 1 can only rely on the assumption of rationality of user 2 to deduce its strategy. For fixed strategy $p_{2}\left(h_{2}\right)$, via Lagrangian duality, the solution of the optimization problem (12) is given by the equation:

$$
\mathbb{E}_{h_{2}}\left[\frac{h_{1}}{\sigma^{2}+p_{1}\left(h_{1}\right) h_{1}+p_{2}\left(h_{2}\right) h_{2}}\right]=\frac{1}{\lambda_{1}}
$$

where $\lambda_{1}$ is chosen in order to satisfy the constraint

$$
\mathbb{E}_{h_{1}}\left[p_{1}\left(h_{1}\right)\right]=\bar{P}_{1} \text {. }
$$

Note that the solution of (13) depends on the power allocation of the second user, which the first user does not know, and reciprocally for the second user. However, given the game model, each user is able to adjust his strategy adaptively to its guess of the strategy of the other user.

For simplicity of presentation, we first consider the scenario of two positive channel states. We then extend it into arbitrary channel states in the end of this section.

\section{A. Two channel states}

In order to simplify the game problem, one assumption has been used in [10] and [11], where the power levels are assumed to be discrete. However, we introduce a slightly different assumption as follows,

\section{Main assumption: channel states are discrete.}

Our main assumption is closely related to the way how feedback channel information are signalled. Once estimated at the receiver side, channel coefficients are feedback to the transmitter with a given precision. Since these are represented on a finite number of bits (e.g. $n$ bits), channels coefficients are mapped on a finite number of $2^{n}$ states. When $n=1$, channel coefficient is coded on 1 bit and may take two possible values (e.g. a representing "bad" channel values, and $b$ representing "good" channel values). When $n=8$, channel coefficient is coded on $2^{8}$ bits. Thus, one can always choose a certain quantization precision that is sufficient to describe a real channel.

Assumption 1: Each carrier's channel gain can be 0 with probability $\rho_{0}$, and can also be other two positive values $a_{1}$ and 
$a_{2}$ (w.l.o.g. $a_{1}<a_{2}$ ) with probability $\rho_{1}$ and $\rho_{2}$ respectively. We have $\rho_{0}+\rho_{1}+\rho_{2}=1$.

Assumption 2: Both users have the same power constraint, define: $\bar{P} \triangleq \bar{P}_{1}=\bar{P}_{2}$. We have

$$
\rho_{1} p_{k}\left(a_{1}\right)+\rho_{2} p_{k}\left(a_{2}\right)=\bar{P}, k=1,2
$$

where $p_{k}\left(a_{1}\right)$ and $p_{k}\left(a_{2}\right)$ represents the power that user $k$ allocates on his carrier when the channel gain is $a_{1}$ and $a_{2}$ respectively. From (15), we have

$$
p_{k}\left(a_{2}\right)=\frac{\bar{P}-\rho_{1} p_{k}\left(a_{1}\right)}{\rho_{2}}
$$

1) Game Formulation: In this part, we focus on the problem of game modeling. We consider a strategic noncooperative game with two players, which completely characterized by three elements as follows,

$$
\Gamma \triangleq\left[\mathcal{K},\left\{\mathcal{P}_{k}\right\}_{k \in \mathcal{K}},\left\{u_{k}\right\}_{k \in \mathcal{K}}\right]
$$

- Player set: $\mathcal{K} \triangleq\{1,2\}$, where $K \triangleq\|\mathcal{K}\|=2$.

- Action set: $\left\{\mathcal{P}_{1}, \mathcal{P}_{2}\right\}$, where $\mathcal{P}_{1} \triangleq\left\{p_{1}\left(a_{1}\right), p_{1}\left(a_{2}\right)\right\}$ and $\mathcal{P}_{2} \triangleq\left\{p_{2}\left(a_{1}\right), p_{2}\left(a_{2}\right)\right\}$. Note that $\mathcal{P}_{k}$ can take any possible value that satisfies power constraint (15).

- Payoff (or utility) function set: $\left\{u_{1}\left(p_{1}^{*}, p_{2}\right), u_{2}\left(p_{1}, p_{2}^{*}\right)\right\}$, where we denote $p_{1}, p_{2}$ as $p_{1}\left(a_{1}\right), p_{2}\left(a_{1}\right)$ for simplifying the following expressions. Note $u_{1}, u_{2}$ are the selfish average capacity as shown in (12). By using (16), we define

$$
\begin{aligned}
u_{1}\left(p_{1}^{*}, p_{2}\right) & =\rho_{1}^{2} \log _{2}\left(1+\frac{a_{1} p_{1}^{*}}{\sigma^{2}+a_{1} p_{2}}\right)+ \\
& +\rho_{1} \rho_{2} \log _{2}\left(1+\frac{a_{2} \frac{\bar{P}-\rho_{1} p_{1}^{*}}{\rho_{2}}}{\sigma^{2}+a_{1} p_{2}}\right)+ \\
& +\rho_{1} \rho_{2} \log _{2}\left(1+\frac{a_{1} p_{1}^{*}}{\sigma^{2}+a_{2} \frac{\bar{P}-\rho_{1} p_{2}}{\rho_{2}}}\right)+ \\
& +\rho_{2}^{2} \log _{2}\left(1+\frac{a_{2} \frac{\bar{P}-\rho_{1} p_{1}^{*}}{\rho_{2}}}{\sigma^{2}+a_{2} \frac{\bar{P}-\rho_{1} p_{2}}{\rho_{2}}}\right) .
\end{aligned}
$$

and

$$
\begin{aligned}
u_{2}\left(p_{1}, p_{2}^{*}\right) & =\rho_{1}^{2} \log _{2}\left(1+\frac{a_{1} p_{2}^{*}}{\sigma^{2}+a_{1} p_{1}}\right)+ \\
& +\rho_{1} \rho_{2} \log _{2}\left(1+\frac{a_{2} \frac{\bar{P}-\rho_{1} p_{2}^{*}}{\rho_{2}}}{\sigma^{2}+a_{1} p_{1}}\right)+ \\
& +\rho_{1} \rho_{2} \log _{2}\left(1+\frac{a_{1} p_{2}^{*}}{\sigma^{2}+a_{2} \frac{\bar{P}-\rho_{1} p_{1}}{\rho_{2}}}\right)+ \\
& +\rho_{2}^{2} \log _{2}\left(1+\frac{a_{2} \frac{\bar{P}-\rho_{1} p_{2}^{*}}{\rho_{2}}}{\sigma^{2}+a_{2} \frac{\bar{P}-\rho_{1} p_{1}}{\rho_{2}}}\right) .
\end{aligned}
$$

In such a non-cooperative game, each player has to maximize its own payoff function, given the other player's strategy and regardless of the consequence his strategy's choice may have on the other player and thus on the overall performance. This means that players play selfishly: they do not communicate before play, and have independent objectives.
2) Nash Equilibrium: A Nash equilibrium for a game is a set of strategies such that no single player can improve its utility by deviating. However, such a equilibrium does not necessarily exist. In our setting, $\left\{p_{1}^{\star}, p_{2}^{\star}\right\}$ is a Nash equilibrium if given any other strategies $p_{1} \in \mathcal{P}_{1}$ and $p_{2} \in \mathcal{P}_{2}$,

$$
\left\{\begin{array}{l}
u_{1}\left(p_{1}^{\star}, p_{2}^{\star}\right) \geq u_{1}\left(p_{1}, p_{2}^{\star}\right) \\
u_{2}\left(p_{1}^{\star}, p_{2}^{\star}\right) \geq u_{2}\left(p_{1}^{\star}, p_{2}\right) .
\end{array}\right.
$$

First, we investigate the existence of an equilibrium in our game model.

Theorem 1: A Nash equilibrium exists in game $\Gamma=$ $\left[\mathcal{K},\left\{\mathcal{P}_{k}\right\}_{k \in \mathcal{K}},\left\{u_{k}\right\}_{k \in \mathcal{K}}\right]$.

The proof of Theorem 1 can be found in appendix A.

Second, we investigate the uniqueness of such an equilibrium in our game model.

Theorem 2: The game $\Gamma=\left[\mathcal{K},\left\{\mathcal{P}_{k}\right\}_{k \in \mathcal{K}},\left\{u_{k}\right\}_{k \in \mathcal{K}}\right]$ has a unique equilibirum.

Proof. From Theorem 1, we know that there exists at least one Nash equilibrium in the game. Let $\mathbf{p}^{\star}=\left\{p_{1}^{\star}, p_{2}^{\star}\right\}$ denote this Nash equilibrium. By definition (19), $\mathbf{p}^{\star}$ has to satisfy $\mathbf{p}^{\star}=\mathbf{r}\left(\mathbf{p}^{\star}\right)$, where $\mathbf{r}\left(\mathbf{p}^{\star}\right)=\left(r_{1}\left(\mathbf{p}^{\star}\right), r_{2}\left(\mathbf{p}^{\star}\right)\right)$, more pricisely, it has to both satisfy the following two equations

$$
\left\{\begin{array}{l}
p_{1}^{\star}=r_{1}\left(p_{2}^{\star}\right)=\max _{p_{1}^{*}} u_{1}\left(p_{1}^{*}, p_{2}^{\star}\right) \\
p_{2}^{\star}=r_{2}\left(p_{1}^{\star}\right)=\max _{p_{2}^{*}} u_{2}\left(p_{1}^{\star}, p_{2}^{*}\right)
\end{array}\right.
$$

Note that $r_{k}\left(p_{-k}^{\star}\right)$ and $r_{k}\left(\mathbf{p}^{\star}\right)$ are equivalent, which are called player $k$ 's best-response function.

Now we assume that Nash equilibrium is not unique and apart from $\mathbf{p}^{\star}$ there exists at least another one, e.g. $\mathbf{p}^{\dagger}=\left\{p_{1}^{\dagger}, p_{2}^{\dagger}\right\}$, and $\mathbf{p}^{\dagger} \neq \mathbf{p}^{\star}$. From definition, it should satisfy (20). However, since utility function $u_{k}$ is concave and differentiable, for a fixed strategy $p_{2}$, there is an unique best strategy $p_{1}^{\prime}$ that satisfies $p_{1}^{\prime}=r_{1}\left(p_{2}\right)$; for a fixed strategy $p_{1}$, there is also an unique best $p_{2}^{\prime}$ that satisfies $p_{2}^{\prime}=r_{1}\left(p_{1}\right)$. So the solution to the equation group (20) is unique and given by equation group

$$
\left\{\begin{array}{l}
\frac{\partial u_{1}}{\partial p_{1}^{*}}=0 \\
\frac{\partial u_{2}}{\partial p_{2}^{*}}=0
\end{array}\right.
$$

It contradicts to our assumption that $\mathbf{p}^{\dagger} \neq \mathbf{p}^{\star}$, therefore, $\mathbf{p}^{\dagger}=$ $\mathbf{p}^{\star}$. We proved Theorem 4

3) Symmetric Game: In this part, we consider the game with the following assumption,

Assumption 3: Both users apply the same power strategy if their observations on channel state(s) are symmetric. Both user do not allocate any power when the carrier's channel gain is 0 .

Note that it is a realistic assumption for many practical reasons. We define

$$
\left\{\begin{array}{l}
p\left(a_{1}\right) \triangleq p_{1}\left(a_{1}\right)=p_{2}\left(a_{1}\right) \\
p\left(a_{2}\right) \triangleq p_{1}\left(a_{2}\right)=p_{2}\left(a_{2}\right)
\end{array}\right.
$$

Now, both users should have an agreement on applying the power allocation strategy $\left\{p\left(a_{1}\right), p\left(a_{2}\right)\right\}$. The decision of applying $p\left(a_{1}\right)$ or $p\left(a_{2}\right)$ only depends on their own channel 
realization. From (15), we have $\rho_{1} p\left(a_{1}\right)+\rho_{2} p\left(a_{2}\right)=\bar{P}$. And we can rewrite the original optimization problem (12) as

$$
\begin{aligned}
\max C_{1}=\max _{p\left(a_{1}\right)} & \rho_{1}^{2} \log _{2}\left(1+\frac{a_{1} p\left(a_{1}\right)}{\sigma^{2}+a_{1} p\left(a_{1}\right)}\right)+ \\
& +\rho_{1} \rho_{2} \log _{2}\left(1+\frac{a_{1} p\left(a_{1}\right)}{\sigma^{2}+a_{2} \cdot \frac{P-\rho_{1} p\left(a_{1}\right)}{\rho_{2}}}\right)+ \\
& +\rho_{1} \rho_{2} \log _{2}\left(1+\frac{a_{2} \cdot \frac{\bar{P}-\rho_{1} p\left(a_{1}\right)}{\rho_{2}}}{\sigma^{2}+a_{1} p\left(a_{1}\right)}\right)+ \\
& +\rho_{2}^{2} \log _{2}\left(1+\frac{a_{2} \cdot \frac{\overline{\bar{P}-\rho_{1} p\left(a_{1}\right)}}{\rho_{2}}}{\sigma^{2}+a_{2} \cdot \frac{\bar{P}-\rho_{1} p\left(a_{1}\right)}{\rho_{2}}}\right) \\
\text { s.t. } \quad & p\left(a_{1}\right) \leq \frac{\bar{P}}{\rho_{1}} \\
& p\left(a_{1}\right) \geq 0
\end{aligned}
$$

In general, the convexity of function $C_{1}\left(p\left(a_{1}\right)\right)$ depends on the relationship between $a_{1}, a_{2}, \rho_{1}, \rho_{2}, \bar{P}$ and $\sigma^{2}$. However, we are more interested in the low noise regime $\left(\sigma^{2} \rightarrow 0\right)$. We define

$$
\begin{aligned}
\tilde{C}_{1}\left(p\left(a_{1}\right)\right) \triangleq & \lim _{\sigma^{2} \rightarrow 0} C_{1}\left(p\left(a_{1}\right)\right) \\
= & \rho_{1}^{2}+\rho_{2}^{2}+\rho_{1} \rho_{2}\left[\log _{2}\left(1+\frac{a_{1} p\left(a_{1}\right)}{a_{2} \cdot \frac{P-\rho_{1} p\left(a_{1}\right)}{\rho_{2}}}\right)+\right. \\
& \left.+\log _{2}\left(1+\frac{a_{2} \cdot \frac{\bar{P}-\rho_{1} p\left(a_{1}\right)}{\rho_{2}}}{a_{1} p\left(a_{1}\right)}\right)\right]
\end{aligned}
$$

Theorem 3: Function $\tilde{C}_{1}\left(p\left(a_{1}\right)\right)$ is decreasing on $\left(0, \frac{a_{2} \bar{P}}{a_{1} \rho_{2}+a_{2} \rho_{1}}\right)$ and increasing on $\left(\frac{a_{2} \bar{P}}{a_{1} \rho_{2}+a_{2} \rho_{1}}, \frac{\bar{P}}{\rho_{1}}\right)$. The solution of the maximization problem $\max _{p\left(a_{1}\right)} \tilde{C}_{1}$ is given by

$$
p^{\star}\left(a_{1}\right)= \begin{cases}0, & a_{2} \rho_{1} \geq a_{1} \rho_{2} \\ \overline{\bar{P}}, & a_{2} \rho_{1}<a_{1} \rho_{2}\end{cases}
$$

which gives the best power strategy

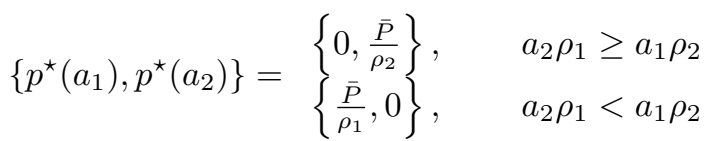

The proof of Theorem 3 can be found in appendix B. Thus, an interesting result is that the choice of the best power strategy depends not only on the channel states but also on the probability that each state happens. In general, the optimal solution will focus all the energy to the channel state that has better condition but with less probability to appear.

\section{B. More channel states}

In the part, we still assume user index $k=1,2$, but each channel gain has $L(L>2)$ possible values. We keep assumption 2 and 3 above, and reclaim assumption 1 as:

Assumption 4: Each carriers channel gain can be 0 with probability $\rho_{0}$, and can also be $L$ positive values $\left\{a_{1}, \ldots, a_{L}\right\}$ (w.l.o.g. $a_{1}<\ldots<a_{L}$ ), each happens with probability $\rho_{1}, \ldots, \rho_{L}$ respectively. We have $\sum_{l=0}^{L} \rho_{l}=1$.

Based on the assumption 3 , we define $p\left(a_{l}\right)$ as

$$
p\left(a_{l}\right) \triangleq p_{1}\left(a_{l}\right)=p_{2}\left(a_{l}\right), l=1, \ldots, L
$$

Now, the power strategy set is $\left\{p\left(a_{1}\right), \ldots, p\left(a_{L}\right)\right\}$. We rewrite the optimization problem (12)

$$
\begin{array}{ll}
\max _{\mathbf{p}} & \sum_{n=1}^{L} \sum_{m=1}^{L} \rho_{m} \rho_{n} \log _{2}\left(1+\frac{a_{n} p\left(a_{n}\right)}{\sigma^{2}+a_{m} p\left(a_{m}\right)}\right) \\
\text { s.t. } & \sum_{l=1}^{L} \rho_{l} p\left(a_{l}\right)=\bar{P} \\
& p\left(a_{l}\right) \geq 0, l=1, \ldots, L
\end{array}
$$

The objective function of this optimization problem is nonconvex. We propose an Zero Interference Searching (ZIS) algorithm as follows,

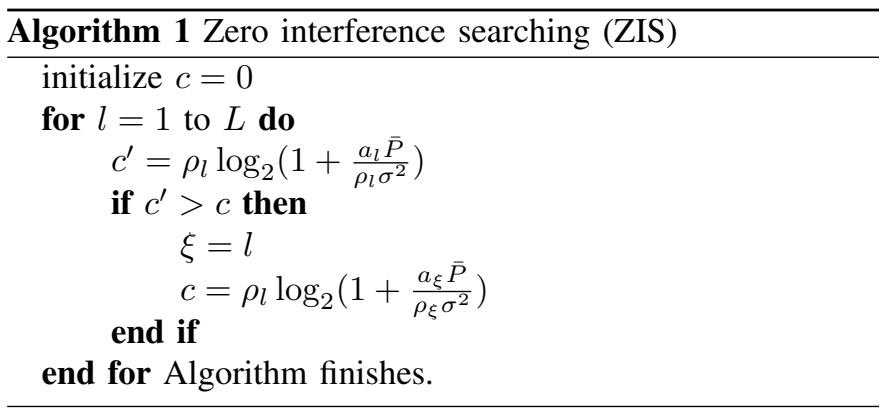

Finally, the output of this algorithm can be shown as $\left(p\left(a_{1}\right), \ldots, p\left(a_{L}\right)\right)=\left(0, \ldots, 0, \frac{P}{\rho_{\xi}}, 0, \ldots, 0\right)$. Each step insists in searching among the cases that all the energy is dedicated to only one best channel state (depends on both $a_{l}$ and $\rho_{l}$ ), where no interference is allowed. The algorithm complexity is $\mathrm{O}(L)$.

\section{NUMERICAL RESUlTS}

In this section, the simulation results are given and analyzed by using the following parameter sets:

\begin{tabular}{|c|c|c|c|c|c|c|}
\hline & $a_{1}$ & $a_{2}$ & $\rho_{1}$ & $\rho_{2}$ & $\bar{P}$ & $\sigma^{2}$ \\
\hline set 1 & 1 & 1.2 & 0.5 & 0.5 & 1 & 0.5 \\
\hline set 2 & 1 & 3 & 0.5 & 0.5 & 1 & 0.1 \\
\hline set 3 & 1 & 10 & 0.5 & 0.5 & 1 & 0.1 \\
\hline
\end{tabular}
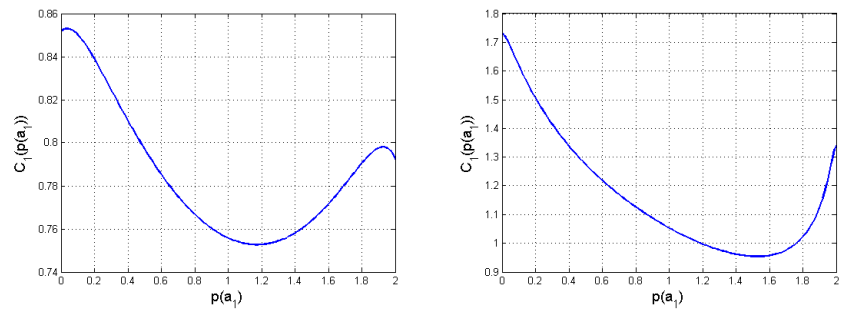

Fig. 2. The non-convexity of function $C_{1}\left(p\left(a_{1}\right)\right)$ (left: set 1 ; right: set 2)

In Fig. 2, we show the behavior of function $C_{1}\left(p\left(a_{1}\right)\right)$ (23) in the symmetric game. In the left figure, we use the parameters from set 1 . We find that function $C_{1}\left(p\left(a_{1}\right)\right)$ is obviously not convex in $p\left(a_{1}\right)$, and the solution $p^{\star}\left(a_{1}\right)$ is comprised between 0 and 0.1 . In the right figure, we use the parameters from set 2 . In this setting, function $C_{1}$ shows the behavior of decreasing and increasing (not convex) and we have $p^{\star}\left(a_{1}\right)=0$. It is consistent with the result of (25) in the case of $a_{2} \rho_{1} \geq a_{1} \rho_{2}$. 

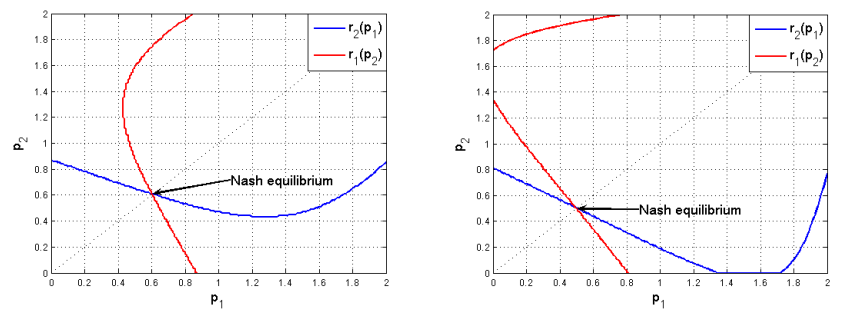

Fig. 3. Illustration of Nash equilibrium (left: set 2; right: set 3)
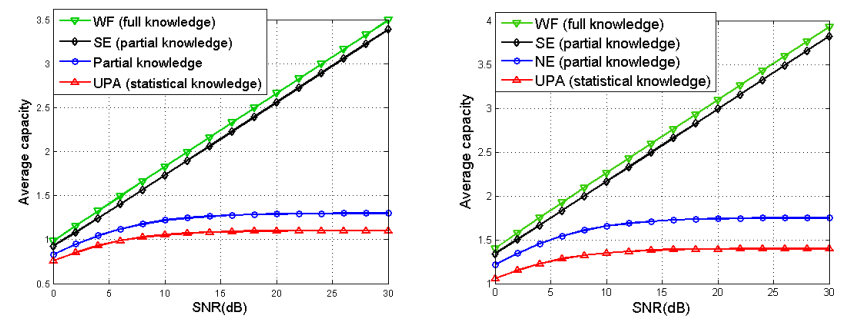

Fig. 4. Average capacity comparison among three different information levels (left: set 2; right: set 3)

In Fig. 3, we show the Nash equilibrium (NE) given by (21). In the left figure, we use the parameters from set 2 ; in the right figure, we use the parameters from set 3 . As expected, there always exists a unique NE for both cases, and it is symmetric, i.e. the NE for set 2 and set 3 are $(0.5,0.5)$ and $(0.6,0.6)$ respectively. It is also interesting to note that when the values of $a_{1}$ and $a_{2}$ are relatively close (set 1 ), the NE is close to the uniform power allocation (UPA), which is $(1,1)$ in this case; when $a_{1}$ and $a_{2}$ are relatively far (set 2), the NE slightly goes away from $(1,1)$.

In Fig. 4, we compare the average capacity by using the parameters from set 2 and set 3 obtained in the three different information level cases considering: complete, partial and statistical knowledge at the transmitter side. The SNR is defined as the ratio between the power constraint $\bar{P}$ and the noise variance $\sigma^{2}$.

- For the case of complete knowledge, recall (9), we have

$$
\lambda \triangleq \lambda_{1}=\lambda_{2}=\frac{1}{3}\left[4 \bar{P}+\sigma^{2}\left(\frac{1}{a_{1}}+\frac{2}{a_{2}}\right)\right],
$$

and the results of waterfilling (WF) in (8) gives the best power strategy (for set 2 and 3 )

$$
\left\{\begin{array}{l}
p_{1}^{\star}\left(a_{1}, a_{1}\right)=p_{2}^{\star}\left(a_{1}, a_{1}\right)=\lambda-\frac{\sigma^{2}}{a_{1}} \\
p_{1}^{\star}\left(a_{2}, a_{2}\right)=p_{2}^{\star}\left(a_{2}, a_{2}\right)=p_{2}^{\star}\left(a_{1}, a_{2}\right)=p_{1}^{\star}\left(a_{2}, a_{1}\right)= \\
=\lambda-\frac{\sigma^{2}}{a_{2}} \\
p_{1}^{\star}\left(a_{1}, a_{2}\right)=p_{2}^{\star}\left(a_{2}, a_{1}\right)=0
\end{array}\right.
$$

- For the case of partial knowledge, the NE is given by equation group (21). In the low noise regime, the symmetric equilibrium (SE) is given by (26). However, in general, if the convexity condition does not hold, since the objective function is differentiable, one can always obtain the best strategy by following the two steps:
- Find all the solutions satisfying $\frac{\partial C_{1}}{\partial p\left(a_{1}\right)}=0$.

- Compare these solutions together with two borders $p\left(a_{1}\right)=0$ and $p\left(a_{1}\right)=\frac{\bar{P}}{\rho_{1}}$, the one that maximizes $C_{1}\left(p\left(a_{1}\right)\right)$ is the best strategy $p^{\star}\left(a_{1}\right)$.

In this simulation, with our parameter settings, the best strategy is $p^{\star}\left(a_{1}\right)=0$ within the range of SNR (0-30dB).

- For the case of statistical knowledge, the best strategy is UPA given by (11).

From Fig. 4, the curves with complete knowledge at Tx provides the highest capacity while the curves with statistical knowledge at Tx provides the lowest. In the case of partial knowledge, the average capacity gain of symmetric equilibrium is higher than Nash equilibrium. Moreover, we find that the efficiency of Nash equilibrium depends on the relationship between $a_{1}$ and $a_{2}$. The efficiency becomes lower/higher when the values of two channel states are close/far.

\section{CONCLUSION}

In the case of uncertain topology when only local information is available, we have provided a power allocation strategy, which depends only on the number of channel states. This strategy was shown to outperform classical uniform power allocation scheme and was not far from the complete CSI in the low SNR regime. Moreover, from a game theoretic view, we have shown the existence and uniqueness of Nash equilibrium. Extension is being studied for the case of MIMO and correlated equilibrium.

\section{APPENDIX}

\section{A. Proof of Theorem 1}

In order to prove Theorem 1, we introduce Theorem 4,

Theorem 4: A Nash equilibrium exists in game $\Gamma=$ $\left[\mathcal{K},\left\{\mathcal{P}_{k}\right\}_{k \in \mathcal{K}},\left\{u_{k}\right\}_{k \in \mathcal{K}}\right]$ if the following two conditions are satisfied, for all $k=1, \ldots, K$ :

1) $\mathcal{P}_{k}$ is a nonempty, convex, and compact subset of some Euclidean space $\mathfrak{R}^{K}$.

2) $u_{k}(\mathbf{P})$ is continuous in $\mathbf{P}$ and quasi-concave in $\mathcal{P}_{k}$.

From (15), we know $p_{1}\left(a_{1}\right), p_{2}\left(a_{1}\right) \in\left[0, \frac{\bar{P}}{\rho_{1}}\right]$, condition 1 is obviously true. For condition 2 , first we prove that $u_{k}$ is a concave function in $\mathcal{P}_{k}$, then we show that any concave function is quasi-concave.

Let $g_{i}\left(p_{k}^{*}\left(a_{1}\right)\right), i=1, \ldots, 4$ be the expressions that inside four log functions in (17). It is easy to see that each $g_{i}(.) \forall i$ is a linear function of $p_{k}^{*}\left(a_{1}\right)$, and so it is concave and also positive. Now, we introduce Theorem 5,

Theorem 5: $f\left(g_{1}(x), \ldots, g_{m}(x)\right)=\sum_{i=1}^{m} \alpha_{i} \log g_{i}(x)$, $\alpha_{i}>0$ is strictly concave in $x$ if $g_{i}$ are strictly concave and positive.

Proof. Function $f$ and $g_{i} \forall i$ are continuous and differentiable. The second derivative of function $f$ is

$$
\frac{\partial^{2} f}{\partial x^{2}}=\alpha_{i}\left[\frac{1}{g_{i}} \frac{\partial^{2} g_{i}}{\partial x^{2}}-\frac{1}{g_{i}^{2}}\left(\frac{\partial g_{i}}{\partial x}\right)^{2}\right]
$$


Since $g_{i}$ are strictly concave and positive, we have $g_{i}>0$ and $\frac{\partial^{2} g_{i}}{\partial x^{2}}<0$. Therefore, we have $\frac{\partial^{2} f}{\partial x^{2}}<0$. So, function $f$ is strict concave in $x$.

From Theorem 5, we showed that $u_{k}$ is a concave function in $\mathcal{P}_{k}$. Then, we give the definition of quasi-concave,

Definition 1: Function $u_{k}: P_{k} \rightarrow \mathfrak{R}$ is quasi-concave if for any $p_{k}, p_{k}^{\prime} \in P_{k}$, we have

$$
u_{k}\left(\lambda p_{k}+(1-\lambda) p_{k}^{\prime}\right) \geq \min \left\{u_{k}\left(p_{k}\right), u_{k}\left(p_{k}^{\prime}\right)\right\}
$$

for all $\lambda \in(0,1)$.

Theorem 6: A concave function is quasi-concave.

Proof. The theorem follows immediately from the observation that if $u_{k}$ is quasi-concave, then for all $p_{k}, p_{k}^{\prime} \in P_{k}$, we have

$$
\begin{aligned}
u_{k}\left(\lambda p_{k}+\right. & \left.(1-\lambda) p_{k}^{\prime}\right) \geq \lambda u_{k}\left(p_{k}\right)+(1-\lambda) u_{k}\left(p_{k}^{\prime}\right) \geq \\
& \geq \min \left\{u_{k}\left(p_{k}\right), u_{k}\left(p_{k}^{\prime}\right)\right\}
\end{aligned}
$$

Function $u_{k}$ is concave and also quasi-concave, therefore, we proved condition 2 in Theorem 4 .

Thus, from Theorem 4-6, we complete the proof for Theorem 1. We can confirm the existence of Nash equilibrium in our game model.

\section{B. Proof of Theorem 3.}

Function $\tilde{C}_{1}\left(p\left(a_{1}\right)\right)$ is continuous and differentiable. From its first derivative

$$
\begin{aligned}
\frac{\partial \tilde{C}_{1}\left(p\left(a_{1}\right)\right)}{\partial p\left(a_{1}\right)}= & \frac{a_{1} a_{2} \rho_{1} \rho_{2}^{2} \bar{P}}{\ln 2\left[a_{1} \rho_{2} p\left(a_{1}\right)+a_{2}\left(\bar{P}-\rho_{1} p\left(a_{1}\right)\right)\right]} . \\
& \cdot\left[\frac{1}{a_{2}\left(\bar{P}-\rho_{1} p\left(a_{1}\right)\right)}-\frac{1}{a_{1} \rho_{2} p\left(a_{1}\right)}\right]
\end{aligned}
$$

We find that it is decreasing on $\left(0, \frac{a_{2} \bar{P}}{a_{1} \rho_{2}+a_{2} \rho_{1}}\right)$ and increasing on $\left(\frac{a_{2} \bar{P}}{a_{1} \rho_{2}+a_{2} \rho_{1}}, \frac{\bar{P}}{\rho_{1}}\right)$. Then we compare $\tilde{C}_{1}(0)$ and $\tilde{C}_{1}\left(\frac{\bar{P}}{\rho_{1}}\right)$ when $\sigma^{2} \rightarrow 0$

$$
\begin{aligned}
& \tilde{C}_{1}(0)=\lim _{\sigma^{2} \rightarrow 0}\left[\rho_{1}^{2}+\rho_{2}^{2}+\rho_{1} \rho_{2} \log _{2}\left(1+\frac{a_{2} \bar{P}}{\rho_{2} \sigma^{2}}\right)\right] \\
& \tilde{C}_{1}\left(\frac{\bar{P}}{\rho_{1}}\right)=\lim _{\sigma^{2} \rightarrow 0}\left[\rho_{1}^{2}+\rho_{2}^{2}+\rho_{1} \rho_{2} \log _{2}\left(1+\frac{a_{1} \bar{P}}{\rho_{1} \sigma^{2}}\right)\right]
\end{aligned}
$$

From (28), we find that when $\frac{a_{2}}{\rho_{2}}>\frac{a_{1}}{\rho_{1}}, \tilde{C}_{1}(0)>\tilde{C}_{1}\left(\frac{\bar{P}}{\rho_{1}}\right)$; when $\frac{a_{1}}{\rho_{1}}>\frac{a_{2}}{\rho_{2}}, \tilde{C}_{1}\left(\frac{\bar{P}}{\rho_{1}}\right)>\tilde{C}_{1}(0)$. Since $a_{1}, a_{2}, \rho_{1}, \rho_{2}$ are positive, we have (25), therefore (26).

\section{REFERENCES}

[1] J. Bingham, "Multicarrier Modulation for Data Transmission: An Idea Whose Time Has Come," in IEEE Communications Magazine, vol. 28, no. 5, pp. 514, May 1990.

[2] R. van Nee and R. Prasad, OFDM for Wireless Multimedia Communications. Boston, USA: Artech House Publishers, 2000.

[3] R. Knopp and P. Humblet, "Information Capacity and Power Control in Single Cell Multi-User Communications," in IEEE Inter. Conf. on Communications, Seattle, WA, pp. 331-335, June 1995.

[4] D. Gesbert and M. S. Alouini, "How Much Feedback is Multi-User Diversity Really Worth?" in IEEE Inter. Conf. on Communications, Paris, France, pp. 234-238, June 2004.

[5] V. Hassel, M. S. Alouini, D. Gesbert, and G. ien, "Exploiting Multiuser Diversity usign Multiple Feedback Thresholds," in Proceedings of the IEEE Vehicular Technology Conference, May 2005.

[6] E. Altman, T. Boulogne, R. E. Azouzi, T. Jimenez, and L. Wynter, "A survey on networking games," Computers and Operations Research, 2005.
[7] L. Lai and H. E. Gamal, "The Water-Filling Game in Fading Multiple Access Channel", IEEE Trans. on Information Theory, 2005.

[8] D. Tse and S. Hanly, "Multiaccess Fading ChannelPart I: Polymatroid Structure, Optimal Resource Allocation and Throughput Capacity," IEEE Trans. on Information Theory, pp. 2796-2815, Nov. 1998.

[9] A. Goldsmith and P. Varaija, "Capacity of Fading Channel with Channel Side Information," IEEE Trans. on Information Theory, Nov. 1997.

[10] E. Altman, K. Avrachenkov, G. Miller, and B. Prabhu, "Discrete Power Control: Cooperative and Non-cooperative Optimization," IEEE Inter. Conf. on Communications, 2007.

[11] S. Adlakha, R. Johari, and A. Goldsmith, "Competition in Wireless Systems via Bayesian Interference Games”, Aug. 2007. 